\title{
Adaptive Control of a Utility-Scale Wind Turbine Operating in Region 3
}

\author{
Susan A. Frost ${ }^{1}$ \\ NASA Ames Research Center \\ Moffett Field, CA 95034 \\ Mark J. Balas ${ }^{2}$ \\ University of Wyoming \\ Laramie, WY 82071 \\ Alan D. Wright ${ }^{3}$ \\ National Renewable Energy Laboratory \\ Golden, CO 80401
}

\begin{abstract}
Adaptive control techniques are well suited to nonlinear applications, such as wind turbines, which are difficult to accurately model and which have effects from poorly known operating environments. The turbulent and unpredictable conditions in which wind turbines operate create many challenges for their operation. In this paper, we design an adaptive collective pitch controller for a high-fidelity simulation of a utility scale, variable-speed horizontal axis wind turbine. The objective of the adaptive pitch controller in Region 3 is to regulate generator speed and reject step disturbances. The control objective is accomplished by collectively pitching the turbine blades. We use an extension of the Direct Model Reference Adaptive Control (DMRAC) approach to track a reference point and to reject persistent disturbances.

The turbine simulation models the Controls Advanced Research Turbine (CART) of the National Renewable Energy Laboratory in Golden, Colorado. The CART is a utility-scale wind turbine which has a well-developed and extensively verified simulator. The adaptive collective pitch controller for Region 3 was compared in simulations with a baseline classical Proportional Integrator (PI) collective pitch controller. In the simulations, the adaptive pitch controller showed improved speed regulation in Region 3 when compared with the baseline PI pitch controller and it demonstrated robustness to modeling errors.
\end{abstract}

\section{Introduction}

$\mathrm{R}$ ated wind speed is the velocity at which maximum power output, or rated power, of a wind turbine is achieved. If a turbine is allowed to operate in an uncontrolled manner, in conditions where the wind speed is above the rated wind speed, the power output would increase in proportion to the cube of the wind speed, resulting in the overheating of the generator and the power electronics system. Additionally, high wind speeds result in larger aerodynamic forces on the machine, possibly leading to system fatigue and failure. Hence, power output of a turbine must be held constant by wind turbine controllers for wind speeds above the turbine's rated wind speed. Region 3 is the area on a plot of turbine power versus wind speed, at and above the rated power point, for which power is held constant ${ }^{1}$.

Turbine power output should be maintained at rated power when operating in Region 3. For a variable-speed machine, a constant torque is applied at the generator, and the turbine rotational speed is maintained at the desired value through the use of blade pitch. In some machines, the pitch angle of each blade is adjusted identically (collective blade pitch control); in others the blade pitch is adjusted independently of the other blades (independent blade pitch control). Collective blade pitch control is a well accepted approach to regulating turbine speed and responding to changes in wind speed ${ }^{2}$. Independent blade pitch control is generally used to compensate for asymmetric wind loads, including vertical wind shear, which we are ignoring.

Adaptive control techniques, ${ }^{3,4}$ are well suited to nonlinear applications, such as wind turbine control. The main nonlinearities in a wind turbine model come from the nonlinear aerodynamic loads on the turbine. In this paper, we focus on the Direct Model Reference Adaptive Control (DMRAC) approach developed in Refs. 5,6. This approach

\footnotetext{
${ }^{1}$ Research Engineer, Intelligent Systems Division, M/S 269-1, AIAA Member.

${ }^{2}$ Professor \& Dept. Head, Department of Electrical and Computer Engineering, 1000 E. University Avenue, AIAA Fellow.

${ }^{3}$ Senior Engineer II, National Wind Technology Center, M/S 3811, 1617 Cole Blvd., AIAA Member.
} 
has the advantage that the reference model and the plant need not have the same dimension; also the DMRAC approach has been extended to handle adaptive rejection of persistent disturbances ${ }^{7,8}$.

The literature suggests that direct adaptive control methods have rarely been used on utility-scale horizontal axis wind turbines (HAWTs). It was shown in Ref. 9 that a pitch controller designed with DMRAC was comparable to a PID pitch controller when regulating turbine speed in a computer simulation of a rigid, nonlinear plant model of the Grumman Windstream-33 HAWT. Adaptive pitch control to optimize power in Region 2 of the Controls Advance Research Turbine (CART) was demonstrated to be effective in simulations and field tests ${ }^{10}$.

In this paper, a DMRAC approach was used to design an adaptive collective pitch controller to operate in conjunction with the Fatigue, Aerodynamics, Structures, and Turbulence Codes (FAST), a high-fidelity simulation of the CART ${ }^{1}$, a utility-scale, variable-speed, horizontal axis wind turbine. The objective of the adaptive pitch controller was to regulate generator speed and to reject step disturbances in Region 3. This objective was accomplished by collectively pitching the turbine blades. It has been demonstrated that the uniform wind disturbance, without shear, across the rotor disk of a turbine can be accurately accounted for when modeled as a step disturbance of unknown amplitude ${ }^{11,12}$. Hence, to improve controller performance we designed the adaptive collective pitch controller to reject step disturbances. The adaptive pitch controller was implemented in the FAST simulation of the CART and tested with step wind inflow and turbulent wind inflow. Modifications to various system parameters were made to simulate modeling errors, thereby testing the robustness of the controllers. Comparisons of the generator speed errors were made between the simulation results of the adaptive pitch controller and the baseline Proportional Integrator (PI) pitch controller implemented for the CART simulation.

\section{CART Configuration and FAST Simulator Specifications}

The CART is a two-bladed, upwind, active-yaw, variable-speed wind turbine located at the National Wind Technology Center (NWTC) in Golden, Colorado. This machine is used as a test bed to study aspects of wind turbine control technology for medium-scale machines ${ }^{1}$. The pitch system on the CART uses electromechanical servos which can pitch the blades up to 18 degrees per second $(\mathrm{deg} / \mathrm{s})$.

In Region 3, the CART uses a conventional variable-speed approach to maintain rated electrical power, which is 600 kilowatts at a low-speed shaft [LSS] speed of 41.7 revolutions per minute (RPM). Power electronics are used to command constant torque from the generator and full-span blade pitch controls the turbine speed.

The FAST Code is a comprehensive aeroelastic simulator capable of predicting both the extreme loads and the fatigue loads of two- and three-bladed horizontal axis wind turbines. FAST uses Kane's method to set up equations of motion, which are solved by numerical integration. FAST uses the AeroDyn subroutine package developed by Windward Engineering to generate aerodynamic forces along the blade ${ }^{13}$. An interface has been developed between FAST and Simulink ${ }^{\circledR}$, enabling users to implement advanced turbine controls in Simulink's block diagram form ${ }^{14}$.

The CART has been modeled with the FAST Codes as a combination of rigid and flexible bodies connected by several degrees of freedom (DOFs). The DOFs can be turned on or off individually for analysis purposes by setting a switch in the input data file. Simulations of the CART running with different controllers are made possible by using the FAST model of the wind turbine with the controller included in the loop.

The FAST code is a well-accepted simulation environment for HAWT's. The FAST code with AeroDyn incorporated in the simulator was evaluated in 2005 by Germanischer Lloyd WindEnergie and found suitable for "the calculation of onshore wind turbine loads for design and certification" ${ }^{15}$. Results from FAST simulations of the CART compared favorably with field tests of similar controllers on the actual CART ${ }^{11}$.

\section{Direct Model Reference Adaptive Control with Rejection of Persistent Disturbances}

In this section, we will develop the theory of the Direct Model Reference Adaptive Control (DMRAC) approach with adaptive rejection of persistent disturbances.

The Plant used in this section of the paper will be modeled by the linear, time-invariant, finite-dimensional system:

$$
\left\{\begin{array}{l}
\dot{\mathbf{x}}_{p}=\mathbf{A x} \mathbf{x}_{p}+\mathbf{B} \mathbf{u}_{p}+\Gamma \mathbf{u}_{D} \\
\mathbf{y}_{p}=\mathbf{C x} \mathbf{x}_{p} ; \mathbf{x}_{p}(0)=\mathbf{x}_{0}
\end{array}\right.
$$


where the plant state, $\mathbf{x}_{p}(t)$, is an $\mathrm{N}_{\mathrm{p}}$-dimensional vector, the control input vector, $\mathbf{u}_{p}(t)$, is M-dimensional, and the sensor output vector, $\mathbf{y}_{p}(t)$, is P-dimensional. The disturbance input vector, $\mathbf{u}_{D}(t)$, is $\mathrm{M}_{\mathrm{D}}$-dimensional and will be thought to come from the Disturbance Generator:

$$
\left\{\begin{array}{l}
\mathbf{u}_{D}=\boldsymbol{\Theta} \mathbf{z}_{D} \\
\dot{\mathbf{z}}_{D}=\mathbf{F} \mathbf{z}_{D} ; \mathbf{z}_{D}(0)=\mathbf{z}_{0}
\end{array}\right.
$$

where the disturbance state, $\mathbf{z}_{D}(t)$, is $\mathrm{N}_{\mathrm{D}}$-dimensional. All matrices in Eqs. (1)-(2) have the appropriate compatible dimensions. Such descriptions of persistent disturbances were first used in Ref. 16 to describe signals of known form but unknown amplitude. Equation (2) can be rewritten in a form that is not a dynamical system, which is sometimes easier to use:

$$
\left\{\begin{array}{l}
\mathbf{u}_{D}=\boldsymbol{\Theta} \mathbf{z}_{D} \\
\mathbf{z}_{D}=\mathbf{L} \phi_{D}
\end{array}\right.
$$

where $\phi_{D}$ is a vector composed of the known basis functions for the solution of $\mathbf{u}_{D}=\Theta \mathbf{z}_{D}$, i.e., $\phi_{D}$ are the basis functions which make up the known form of the disturbance, and $\mathbf{L}$ is a matrix of dimension $\mathbf{N}_{D} \times \operatorname{dim}\left(\phi_{D}\right)$. The method for rejecting persistent disturbances used in this paper requires only the knowledge of the form of the disturbance, the amplitude of the disturbance does not need to be known, i.e. $(\mathbf{L}, \Theta)$ can be unknown. In this paper, we will be interested in rejecting step disturbances of unknown amplitude which can be represented in the form of Eq. (3) as $\phi_{D} \equiv 1$, with $(\mathbf{L}, \Theta)$ unknown.

In Ref. 7, as with much of the control literature, it is assumed that the plant and disturbance generator parameter matrices, $(\mathbf{A}, \mathbf{B}, \mathbf{C}, \Gamma, \Theta, \mathbf{F})$, are known. This knowledge of the plant and its disturbance generator allows the Separation Principle of Linear Control Theory to be invoked to arrive at a State-Estimator based, linear controller which can suppress the persistent disturbances via feedback. In this paper, we will not assume that the plant and disturbance generator parameter matrices, $(\mathbf{A}, \mathbf{B}, \mathbf{C}, \Gamma, \Theta)$, are known. But, we will assume that the disturbance generator parameter from Eq. (2), F, is known, i.e., the form of the disturbance functions is known. In many cases, knowledge of $\mathbf{F}$ is not a severe restriction, since the disturbance function is often of known form but unknown amplitude.

Our control objective will be to cause the output of the plant, $\mathbf{y}_{p}(t)$, to asymptotically track the output of a known reference model, $\mathbf{y}_{m}(t)$. The Reference Model is given by

$$
\left\{\begin{array}{l}
\dot{\mathbf{x}}_{m}=\mathbf{A}_{m} \mathbf{x}_{m}+\mathbf{B}_{m} \mathbf{u}_{m} ; \mathbf{x}_{m}(\mathbf{0})=\mathbf{x}_{0}^{m} \\
\mathbf{y}_{m}=\mathbf{C}_{m} \mathbf{x}_{m}
\end{array}\right.
$$

where the reference model state, $\mathbf{x}_{m}(t)$, is an $\mathrm{N}_{\mathrm{m}}$-dimensional vector. The reference model output, $\mathbf{y}_{m}(t)$, must have the same dimension as the plant output, $\mathbf{y}_{p}(t)$. The excitation of the reference model is accomplished via the vector, $\mathbf{u}_{m}(t)$, which is generated by

$$
\dot{\mathbf{u}}_{m}=\mathbf{F}_{m} \mathbf{u}_{m} ; \mathbf{u}_{m}(0)=\mathbf{u}_{0}^{m}
$$

It is assumed that the reference model is stable and the model parameters, $\left(\mathbf{A}_{m}, \mathbf{B}_{m}, \mathbf{C}_{m}, \mathbf{F}_{m}\right)$, are known.

The desired control objective is for the output of the plant to asymptotically track the output of the reference model. We define the output error vector as: 


$$
\mathbf{e}_{y} \equiv \mathbf{y}_{p}-\mathbf{y}_{m}
$$

To achieve the desired control objective, we want $\mathbf{e}_{y} \underset{t \rightarrow \infty}{\longrightarrow} 0$.

Consider the plant given by Eq. (1) with the disturbance generator given by Eq. (3). Our control objective for this system will be accomplished by an Adaptive Control Law of the form:

$$
\mathbf{u}_{p}=\mathbf{G}_{m} \mathbf{x}_{m}+\mathbf{G}_{u} \mathbf{u}_{m}+\mathbf{G}_{e} \mathbf{c}_{y}+\mathbf{G}_{D} \phi_{D}
$$

where $\mathbf{G}_{m}, \mathbf{G}_{u}, \mathbf{G}_{e}$, and $\mathbf{G}_{D}$ are matrices of the appropriate compatible dimensions, whose definitions will be given later. In a previous paper ${ }^{8}$, we developed the gain adaptation laws to make asymptotic output tracking possible.

Now we specify the Adaptive Gain Laws, which will produce asymptotic tracking:

$$
\left\{\begin{array}{l}
\dot{\mathbf{G}}_{u}=-\mathbf{e}_{y} \mathbf{u}_{m}^{T} \mathbf{h}_{11} \\
\dot{\mathbf{G}}_{m}=-\mathbf{e}_{y} \mathbf{x}_{m}^{T} \mathbf{h}_{22} \\
\dot{\mathbf{G}}_{e}=-\mathbf{e}_{y} \mathbf{e}_{y}^{T} \mathbf{h}_{33} \\
\dot{\mathbf{G}}_{D}=-\mathbf{e}_{y} \phi_{D}^{T} \mathbf{h}_{44}
\end{array}\right.
$$

where $\mathbf{h}_{i i}, i=1,2, \ldots, 4$ are arbitrary, positive definite matrices. Our Adaptive Controller is specified by Eq. (8) with the above adaptive gain laws Eq. (9). See Ref. 8 for an analysis of the stability of this controller and the proof of the following result:

Suppose the following are true:

(1) All $\mathbf{u}_{m}(t)$ are bounded (i.e., all eigenvalues of $\mathbf{F}_{m}$ are in the closed left-half plane);

(2) The reference model (4) is stable (i.e., all eigenvalues of $\mathbf{A}_{m}$ are in the open left-half plane);

(3) $\phi_{D}$ is bounded ( i.e., all eigenvalues of $\mathbf{F}$ are in the closed left-half plane and any eigenvalues on the imaginary axis are simple);

(4) (A, B, C) is Almost Strict Positive Real (ASPR), i.e., $\mathbf{T}_{C}(s) \equiv \mathbf{C}\left(s \mathbf{I}-\mathbf{A}_{C}\right)^{-1} \mathbf{B}$ is strictly positive real.

Then the adaptive gains, $\mathbf{G}_{u}, \mathbf{G}_{m}, \mathbf{G}_{e}$, and $\mathbf{G}_{D}$, remain bounded and asymptotic tracking occurs, i.e. $\mathbf{e}_{y} \equiv \mathbf{y}_{p}-\mathbf{y}_{m} \longrightarrow \underset{t \rightarrow \infty}{\longrightarrow}$.

\section{Adaptive Collective Pitch Controller for a Simulation of the CART}

The plant we used in the FAST simulations of the CART was a simple wind turbine model with turbine aerodynamics included along with the generator degree of freedom. The plant neglected all other dynamics and degrees of freedom. The FAST Codes were used to model the nonlinear aerodynamic loads on the turbine in our plant. A state space model of the plant was generated by the FAST Codes, see Refs. 14, 17 to obtain the model. The output of the plant was the generator speed. The control input was the collective pitch angle commands.

A classical PI collective pitch controller (the baseline PI pitch controller) has been implemented and tested in the FAST simulation of the CART. A similar version of the baseline PI pitch controller has been tested on the actual CART. The baseline PI pitch controller in the FAST simulator provides a basis for comparison with the adaptive pitch controller.

The control goal of a collective pitch controller for a wind turbine operating in Region 3 is to regulate generator speed using blade pitch. The FAST simulator model of the CART measures generator speed and feeds it back to the baseline PI pitch controller which regulates it to the rated generator speed for the wind turbine. The baseline PI pitch controller accomplishes the regulation of the generator speed by collectively pitching the turbine blades. 
The adaptive collective pitch controller that we designed for this paper replaces the baseline PI pitch controller in the FAST simulator, see Fig. 1. The generator speed was measured and fed back to the adaptive controller, as it was in the baseline PI pitch controller. The adaptive pitch controller was designed with the DMRAC approach described in section III, with the control objective of regulating generator speed and rejecting persistent step disturbances. The step disturbances account for wind speed fluctuations and wind gusts that the turbine may experience during operation. Our control objective was accomplished by an Adaptive Control Law of the form given in Eq. (8) with gains specified in Eq. (9):

$$
\mathbf{u}_{p}=\mathbf{G}_{m} \mathbf{x}_{m}+\mathbf{G}_{u} \mathbf{u}_{m}+\mathbf{G}_{e} \mathbf{e}_{\boldsymbol{y}}+\mathbf{G}_{D} \phi_{D}
$$

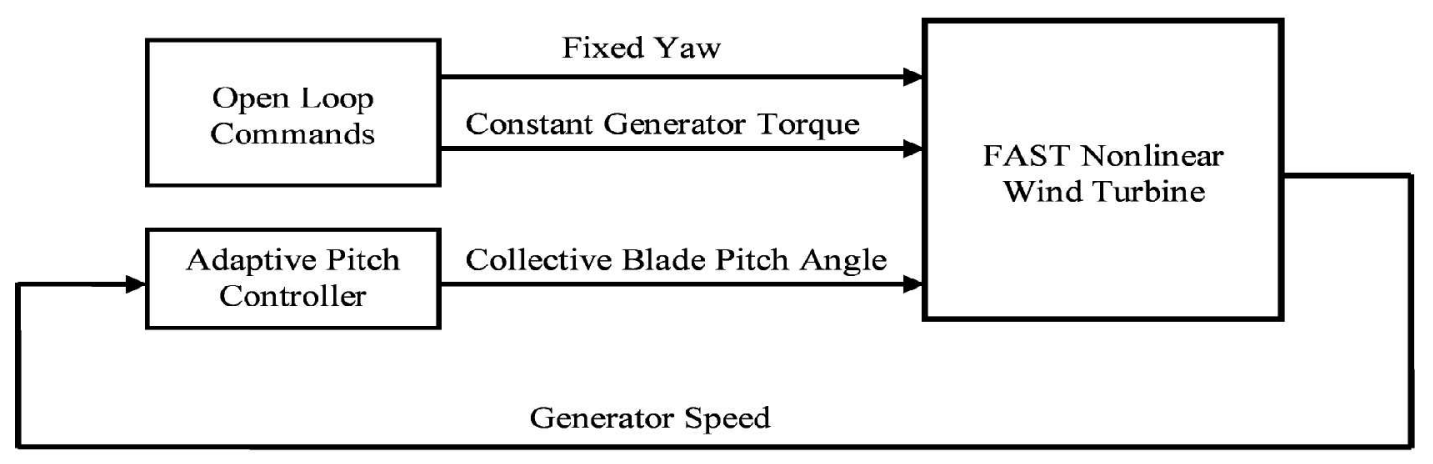

Figure 1. Block diagram of adaptive pitch controller.

Since our objective is to regulate generator speed, there is no need to track a reference model, therefore, $\mathbf{G}_{m}$ and $\mathbf{G}_{u}$ are identically zero in Eq. (10) above. The disturbance created by fluctuations in wind speed acting on a turbine operating in Region 3 has been shown to be well-modeled by a step function of unknown amplitude ${ }^{11,12}$. For a step function, the disturbance generator function in the form of Eq. (3) is specified by $\phi_{D}=1$. Recall that we are not required to know the amplitude of the disturbance function, i.e., (L, $(\mathbf{)})$ from Eq. (3) need not be known. Now, the adaptive control law which accomplishes the control goals described above is:

$$
\left\{\begin{array}{l}
\mathbf{u}_{p}=\mathbf{G}_{e} \mathbf{e}_{y}+\mathbf{G}_{D} \\
\dot{\mathbf{G}}_{e}=-\mathbf{e}_{y} \mathbf{e}_{y}^{T} \mathbf{h}_{33} \\
\dot{\mathbf{G}}_{D}=-\mathbf{e}_{y} \mathbf{h}_{44}
\end{array}\right.
$$

where $\mathbf{h}_{33}, \mathbf{h}_{44}$ are arbitrary, positive definite matrices.

The adaptive controller specified by Eq. (11) was implemented in Simulink in the FAST simulation of the CART. The adaptive controller gains, $\mathbf{h}_{33}$ and $\mathbf{h}_{44}$, were tuned to minimize the generator speed error, since we had the goal of regulating generator speed, while keeping the blade pitch rate in a range similar to that of the baseline PI controller. The actual values of the gains used in the adaptive controller were: $h_{33}=14$ and $h_{44}=0.5$.

\section{Simulation Conditions and Results}

The FAST simulations of the CART were run from time 0 seconds to 100 seconds with an integration step size of 0.006 seconds. The generator DOF switch was turned on. The other DOF switches were turned off. The wind turbine had fixed-yaw with no yaw control. Aerodynamic forces were calculated during the runs. The parametric information for the FAST simulator as we configured it is available from Refs. 14, 17.

Simulations were run with two types of wind inflow to the FAST simulator: step wind and turbulent wind. Comparisons were made between the baseline PI pitch controller and the adaptive pitch controller under the 
conditions of the two wind inflows. We evaluated the generator speed errors of each controller, since our objective was to regulate generator speed. The generator speed error is the absolute value of the difference between the generator speed and the rated generator speed, which is 1800 RPM for the CART operating in Region 3. A normalized generator speed error for each simulation was calculated by taking the square root of the sum of the squares of the generator errors and dividing by the total number of errors.

All figures related to the simulation start at time 20 seconds, once the transients due to system start-up have died off. The first simulations described in this paper had step wind inflow, see Fig. 2a. All of the step wind inflow results in Region 3 turbine operation. The generator speed error for the baseline PI pitch controller and the adaptive pitch controller was calculated at each time step and the results are shown in Fig. 2b. For the step wind, the PI controller had a normalized generator error of 0.0922 RPM and the adaptive controller had a normalized generator error of 0.0479 RPM. The relative difference between the errors is $48.0 \%$. The adaptive pitch controller had more pitch activity than the PI controller; but the magnitude of the blade pitch rates from the PI controller and the adaptive controller are comparable and are well below the pitch rate limits of $\pm 18 \mathrm{deg} / \mathrm{s}$ that are implemented in the CART.
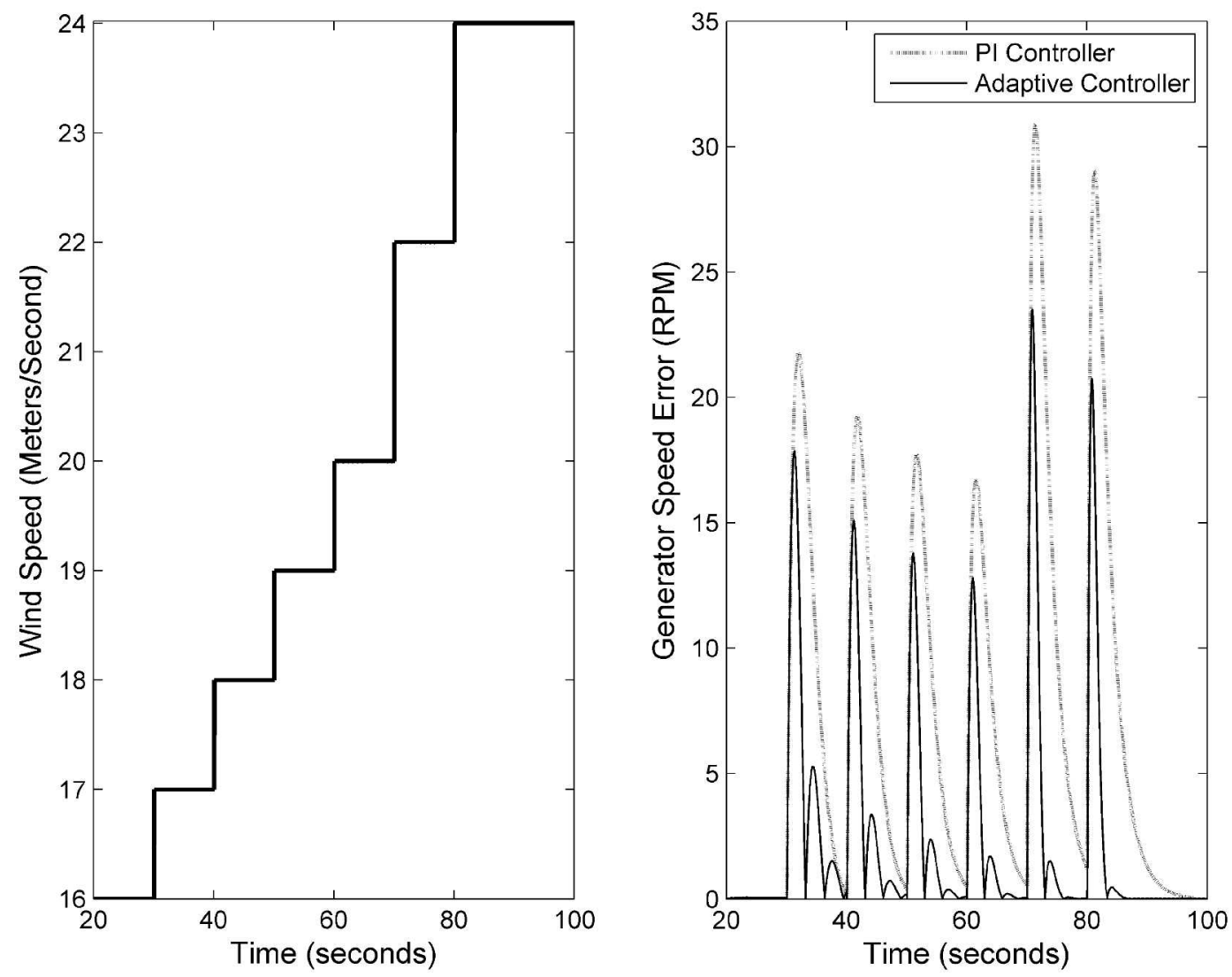

Figure 2. (a) Step wind inflow to simulation and (b) generator speed errors for baseline PI and adaptive pitch controllers with step wind inflow.

A turbulent wind inflow to the FAST simulator was used in the second set of simulations, see Fig. 3a. The majority of the turbulent wind inflow resulted in Region 3 turbine operation. The generator speed errors for the baseline PI pitch controller and the adaptive pitch controller were calculated at each time step, see Fig. 3b. When the simulation was run with turbulent wind inflow, the baseline PI pitch controller had a normalized generator error of 0.2435 RPM and the adaptive controller had a normalized generator error of 0.0781 RPM. The relative difference between the errors was $67.9 \%$. The magnitude and pitch rate activity of the PI controller and the adaptive controller were comparable during the simulations with turbulent wind inflow. 

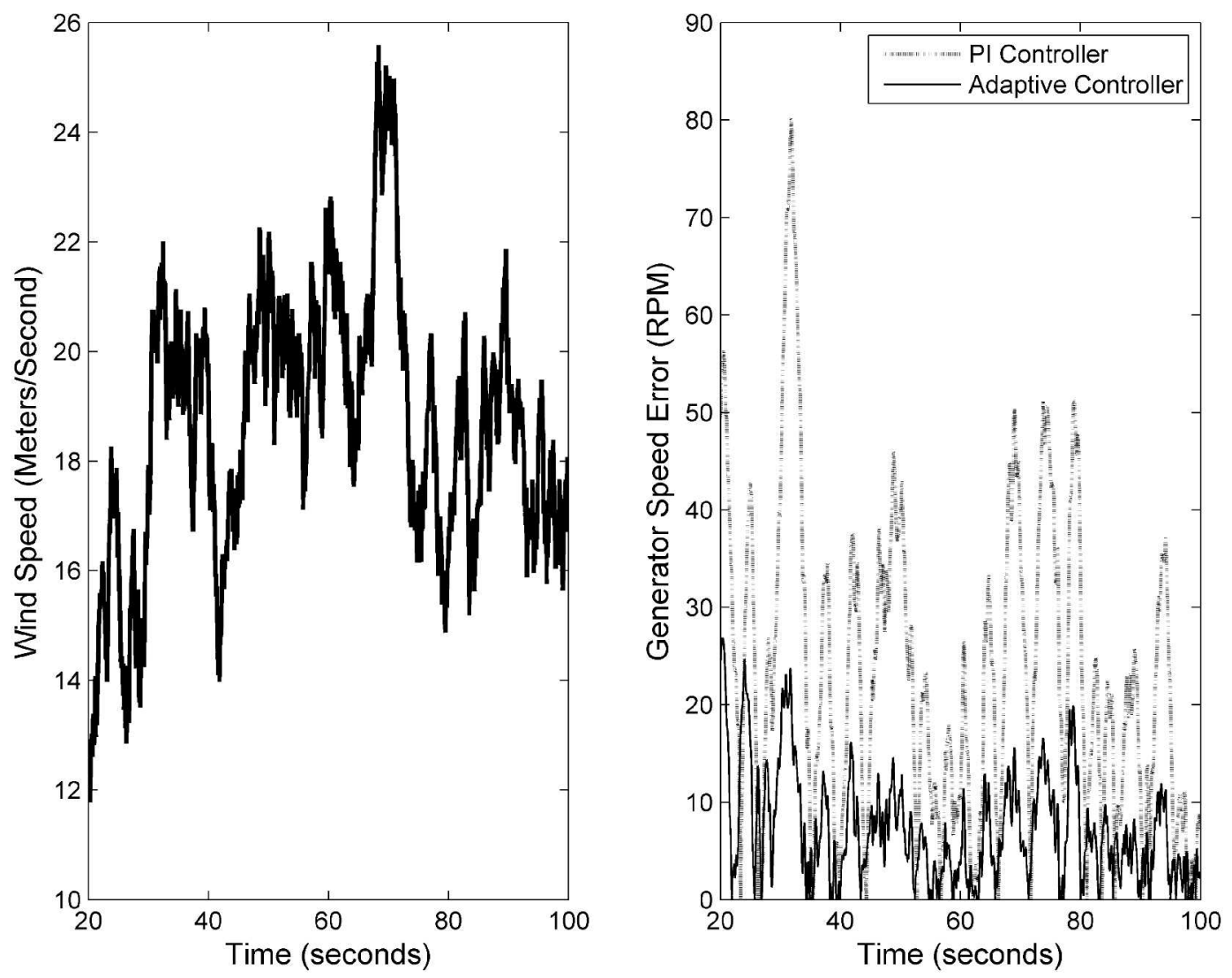

Figure 3. (a) Turbulent wind inflow to simulation and (b) generator speed errors for baseline PI and adaptive pitch controllers with turbulent wind inflow.

Additional simulations were run in which modifications were made to various parameters affecting the modeling of the CART. Some of the parameter modifications made included: varying the aerodynamic twist properties of the turbine blade, increasing the blade chord length, and turning on the drive-train rotational flexibility DOF. Both controllers performed in a robust manner in the presence of these parameter variations.

Future work will include running tests on the actual CART with the adaptive pitch controller replacing the baseline PI pitch controller. Further investigation of parameter modifications will be performed, such as adding a third blade to the wind turbine, which would require redesign of the baseline PI controller but no redesign of the adaptive controller. We would also like to exploit the ability of the DMRAC method developed in this paper to track a reference model. Different tracking models representing an ideal wind turbine could be used in the design of an adaptive pitch controller and tested in simulations.

\section{Conclusions}

An adaptive collective pitch controller using DMRAC with rejection of step disturbances of unknown amplitude showed promise for improved generator speed regulation in simulations of the CART. The adaptive pitch controller reduced the generator speed errors when compared with the baseline PI pitch controller in simulations of Region 3 operation with both step wind and turbulent wind inflow. The adaptive pitch controller demonstrated robustness to modeling errors without any modifications to the controller.

\section{References}

${ }^{1}$ Fingersh, LJ, Johnson, KE. Baseline results and future plans for the NREL Controls Advance Research Turbine. Proceedings of the $23^{\text {rd }}$ AIAA Aerospace Sciences Meeting and Exhibit Wind Energy Symposium 2004; 87-93.

${ }^{2}$ Wright, AD, Stol, KA, Fingersh, LJ. Progress in implementing and testing state-space controls for the controls advanced research turbine. Proceedings of the 24 $4^{\text {th }}$ AIAA Aerospace Sciences Meeting and Exhibit Wind Energy Symposium 2005; 88-100.

${ }^{3}$ Narendra, KS, Annaswamy AM. Stable Adaptive Systems. Prentice-Hall: New Jersey, 1989. 
${ }^{4}$ Åström, KJ, Wittenmark, B. Adaptive Control ( $2^{\text {nd }}$ edition). Addison-Wesley: Massachusetts, 1995.

${ }^{5}$ Wen, JT, Balas, MJ. Robust adaptive control in Hilbert space. Journal of Mathematical Analysis and Application 1989; 143(1): 1-26.

${ }^{6}$ Balas, MJ. Finite-dimensional direct adaptive control for discrete-time infinite-dimensional linear systems. Journal of Mathematical Analysis and Applications 1995; 196(1): 153-171.

${ }^{7}$ Fuentes, RJ, Balas, MJ. Direct adaptive rejection of persistent disturbances. Journal of Mathematical Analysis and Applications 2000; 251(1): 28-39.

${ }^{8}$ Frost, SA, Balas, MJ, and Wright, AD. International Journal of Robust and Nonlinear Control, DOI: 10.1002/mc.1329.

${ }^{9}$ Freeman, JB, Balas, MJ. Direct model-reference adaptive control of variable speed horizontal-axis wind turbines. Wind Engineering 1998; 22: 209-218.

${ }^{10}$ Johnson, KE, Fingersh, LJ. Adaptive pitch control of variable-speed wind turbines. Collection of Technical Papers $-45^{\text {th }}$ AIAA Aerospace Sciences Meeting and Exhibit 2007; 18:12391-12398.

${ }^{11}$ Wright, AD, Balas, MJ, Fingersh, LJ. Testing state-space controls for the controls advanced research turbine. Transactions of the ASME. Journal of Solar Energy Engineering 2006; 128(4): 506-515.

${ }^{12}$ Wright, AD, Balas, MJ. Design of controls to attenuate loads in the controls advanced research turbine. Transactions of the ASME. Journal of Solar Energy Engineering 2004; 126(4): 1083-91.

${ }^{13}$ Laino, DJ, Hansen, AC. User's guide to the computer software routines AeroDyn interface for ADAMSß. Salt Lake City, Utah: Windward Engineering, LC, September 2001.

${ }^{14}$ Jonkman, JM, Buhl, ML. FAST user's guide, National Renewable Energy Laboratory, NREL/EL-500-38230, Golden, Colorado, August, 2005.

${ }^{15}$ Manjock, A. Evaluation report: Design codes FAST and ADAMS for load calculations of onshore wind turbines. Report No. 72042. Hamburg, Germany: Germanischer Lloyd WindEnergie GmbH, May 25, 2005.

${ }^{16}$ Johnson, C.D. Theory of disturbance-accommodating controllers. Control \& Dynamic Systems, Advances in Theory and Applications, Leondes, CT. ed. Academic Press: New York, 1976; 12: 387-489.

${ }^{17}$ National Renewable Energy Lab., NWTC Design Codes (FAST), http://wind.nrel.gov/designcodes/simulators/fast/, National Renewable Energy Lab., Golden, CO, May 26, 2008. 